\title{
Automated Quantitative Pupillometry for the Prognostication of Coma After Cardiac Arrest
}

\author{
Tamarah Suys $\cdot$ Pierre Bouzat $\cdot$ Pedro Marques-Vidal $\cdot$ \\ Nathalie Sala $\cdot$ Jean-François Payen • \\ Andrea O. Rossetti · Mauro Oddo
}

Published online: 24 April 2014

(c) Springer Science+Business Media New York 2014

\begin{abstract}
Background Sedation and therapeutic hypothermia (TH) delay neurological responses and might reduce the accuracy of clinical examination to predict outcome after cardiac arrest (CA). We examined the accuracy of quantitative pupillary light reactivity (PLR), using an automated infrared pupillometry, to predict outcome of post-CA coma in comparison to standard PLR, EEG, and somato-sensory evoked potentials (SSEP).

Methods We prospectively studied over a 1-year period (June 2012-June 2013) 50 consecutive comatose CA patients treated with $\mathrm{TH}\left(33^{\circ} \mathrm{C}, 24 \mathrm{~h}\right)$. Quantitative PLR
\end{abstract}

Tamarah Suys and Pierre Bouzat contributed equally.

T. Suys $\cdot$ P. Bouzat $\cdot$ N. Sala $\cdot$ M. Oddo $(\bowtie)$

Neuroscience Critical Care Research Group, Department of Intensive Care Medicine, Centre Hospitalier Universitaire Vaudois (CHUV), University Hospital and Faculty of Biology and Medicine, University of Lausanne, Rue du Bugnon 46, 1011 Lausanne, Switzerland

e-mail: mauro.oddo@chuv.ch

P. Bouzat $\cdot$ J.-F. Payen

Department of Anesthesiology and Intensive Care Medicine, Grenoble University Hospital and Joseph Fourier University, Grenoble, France

P. Marques-Vidal

Department of Internal Medicine, Centre Hospitalier Universitaire Vaudois (CHUV), University Hospital and Faculty of Biology and Medicine, University of Lausanne, Lausanne, Switzerland

\section{A. O. Rossetti}

Department of Clinical Neurosciences, Centre Hospitalier Universitaire Vaudois (CHUV), University Hospital and Faculty of Biology and Medicine, University of Lausanne, Lausanne, Switzerland (expressed as the $\%$ of pupillary response to a calibrated light stimulus) and standard PLR were measured at day 1 (TH and sedation; on average $16 \mathrm{~h}$ after $\mathrm{CA}$ ) and day 2 (normothermia, off sedation: on average $46 \mathrm{~h}$ after CA). Neurological outcome was assessed at 90 days with Cerebral Performance Categories (CPC), dichotomized as good (CPC 1-2) versus poor (CPC 3-5). Predictive performance was analyzed using area under the ROC curves (AUC).

Results Patients with good outcome $[n=23(46 \%)]$ had higher quantitative PLR than those with poor outcome $[n=27 ; 16$ (range $9-23)$ vs. $10(1-30) \%$ at day 1 , and 20 (13-39) vs. $11(1-55) \%$ at day 2 , both $p<0.001]$. Best cut-off for outcome prediction of quantitative PLR was $<13 \%$. The AUC to predict poor outcome was higher for quantitative than for standard PLR at both time points (day $1,0.79$ vs. $0.56, p=0.005$; day $2,0.81$ vs. 0.64, $p=0.006$ ). Prognostic accuracy of quantitative PLR was comparable to that of EEG and SSEP (0.81 vs. 0.80 and 0.73 , respectively, both $p>0.20$ ).

Conclusions Quantitative PLR is more accurate than standard PLR in predicting outcome of post-anoxic coma, irrespective of temperature and sedation, and has comparable prognostic accuracy than EEG and SSEP.

Keywords Cardiac arrest - Therapeutic hypothermia . Outcome - Coma - Prognosis · Pupillometry .

Pupillary light reactivity $\cdot$ Pupillary response $\cdot$ EEG .

Evoked potentials

\section{Introduction}

Implementation of post-resuscitation care and therapeutic hypothermia $(\mathrm{TH})$ has significantly increased the number of 
patients surviving from prolonged cardiac arrest (CA) with a good neurological recovery $[1,2]$. TH and comprehensive post-resuscitation care is expensive and resource intensive. Patients who will have good recovery are currently often indistinguishable from patients who will eventually have a poor prognosis. In this context, adequate prognostication of neurological outcome in the early phase following CA is of great importance, particularly because it may allow appropriate allocation of resources [3]. The initial assessment of prognosis in post-CA comatose patients is primarily based on neurological examination [4]. However, sedation and hypothermia delay drug elimination and alter clinical tests $[5,6]$. In the setting of $\mathrm{TH}$, standard neurological examination (including motor response and brainstem reflexes) may not always be accurate to predict neurological recovery [7-9]. The addition of electroencephalography (EEG) [912] and somato-sensory evoked potentials (SSEP) [13, 14] might significantly improve prognostication of post-CA coma. However, EEG and SSEP are not always available and require specific expertise for their interpretation [15]. In this setting, the ideal tool would be a simple, widely available, and quantitative technique.

Automated video pupillometry is a novel electronic device that contains an infrared light camera which enables to measure quantitatively the percentage of pupillary reaction to a calibrated light stimulation. In critically ill patients, two preliminary studies found that the measurement of pupillary size with an automated pupillometer was more accurate than standard pupillary examination using a manual pen light [16, 17]. Yan et al. [18], in a larger cohort of liver transplant patients, found that peri-operative pupillary abnormalities measured with quantitative pupillometry were associated with neurological complications. Behrends et al. [19], in a small cohort of 30 patients, found that quantitative pupillary reactivity performed during cardio-pulmonary resuscitation was associated with early survival. No studies have examined the potential value of automated pupillometry in predicting neurological outcome of comatose critically ill patients in general and of comatose post-CA patients in particular. The objective of this prospective observational double-blinded study was to evaluate whether automated quantitative pupillometry had higher accuracy than standard pupillary examination in predicting the outcome of post-CA coma, and to compare its prognostic accuracy to that of electro-physiological exams, including EEG and SSEP.

\section{Methods}

\section{Patients}

Subjects were part of an ongoing prospective outcome database of comatose CA patients, successfully resuscitated from an out-of-hospital CA and admitted to the medical/surgical ICU of the Lausanne University Hospital, Lausanne, Switzerland, over a 1-year period. Approval for the study was given by the Ethical Committee of the University of Lausanne. The population consisted of patients older than 16 years admitted for coma following out-of-hospital CA between June 2012 and June 2013, treated with $\mathrm{TH}$.

\section{General Management}

Patients were admitted to the ICU where TH was applied following a standardized written institutional algorithm, as recently described [20-22]. TH was considered in adults with cardiac and non-cardiac etiologies of CA, regardless of the initial CA rhythm, unless presenting severe hemodynamic instability, or a patient's "do not resuscitate" order [7, 23].

Cooling was induced immediately on hospital admission with ice-cold packs and intravenous ice-cold fluids. Subsequently, TH was maintained at $33 \pm 1{ }^{\circ} \mathrm{C}$ for $24 \mathrm{~h}$ using a surface cooling device with a computerized adjustment of patient temperature target (Arctic Sun 2200 TTM $^{\circledR}$, Bard Medical, Louisville, CO, USA). Sedation (midazolam, $0.1 \mathrm{mg} / \mathrm{kg} / \mathrm{h}$ ), analgesia (fentanyl, $1.5 \mu \mathrm{g} / \mathrm{kg} / \mathrm{h}$ ) and neuromuscular blocking agents (rocuronium $0.6 \mathrm{mg} / \mathrm{kg}$ bolus, if shivering) were administered intravenously during $\mathrm{TH}$, according to a written standardized algorithm, and were discontinued after passive rewarming, once core temperature was above $35^{\circ} \mathrm{C}$.

Patients were mechanically ventilated to target $\mathrm{PaCO}_{2}$ between 35 and $45 \mathrm{mmHg}$ and $\mathrm{PaO}_{2}$ of $80-100 \mathrm{mmHg}$. Mean arterial pressure was maintained $>70 \mathrm{mmHg}$ with volume resuscitation (mainly isotonic solutions) and norepinephrine when needed.

\section{Measure of Pupillary Reactivity}

\section{Measure of Standard Pupillary Light Reactivity}

Standard pupillary light reactivity (PLR) was measured manually using a standard pen light by the ICU nurse in charge of the patient, according to our algorithm and standard practices of care. Standard PLR was coded qualitatively as absent (no reactivity observed) or present when reactivity was observed.

\section{Measure of Quantitative Pupillary Light Reactivity}

The NeuroLight Algiscan ${ }^{\circledR}$ (IDMED, Marseille, France) is a video automated pupillometer that allows the measure of PLR quantitatively. This portable pupillometer device contains an infrared camera that enables the video registration of 
the variation of the pupillary surface. It integrates a calibrated light stimulation ( 320 Lux, $1 \mathrm{~s}$ ) that allows the rapid and precise measurement $(\approx 0.05 \mathrm{~mm}$ limit) of patient PLR. Baseline pupil size (expressed in $\mathrm{mm}$ ) and the quantitative PLR (expressed as the percentage of pupillary light response) are measured within $4 \mathrm{~s}$ from the start of a calibrated light stimulation. Measure of quantitative PLR was conducted by the ICU research nurse (TS) and was performed in triplicates for each eye at two time points: at day 1 after CA, during TH and under sedation-analgesia and at day 2, after CA, following TH and rewarming, in normothermic conditions, off sedation, and analgesia. At each time point, the best value was retained for the analysis.

\section{Electrophysiological Tests}

Video-EEGs (Viasys Neurocare, Madison, WI, USA) were performed at day 1 , during $\mathrm{TH}$, and at day 2 , in normothermia, using 21 electrodes according to the international 10-20 system. Background reactivity was tested at the bedside, as previously reported, by applying repetitive auditory, visual, and nociceptive stimuli [7, 23]. EEG findings were categorized by certified EEG interpreters according to the presence/absence of background reactivity, defined as an activity of $\geq 10 \mu \mathrm{V}$ (regardless of frequency) with any clear and reproducible change in amplitude or frequency upon stimulation, excluding "stimulus induced rhythmic, periodic, or ictal discharges" (SIRPIDS) and muscle artifacts [7, 10].

The cortical N20 responses on SSEP, performed at day 2 in normothermia and off sedation, were categorized as present or bilaterally absent, defined as no clear negative deflection at $18-25 \mathrm{~ms}$ followed by a positive wave on both sides.

\section{Study Design}

The study was prospective and double-blinded, i.e., the neurologists who performed electrophysiological tests and assessed patient outcome were blinded to quantitative PLR, and the research ICU nurse who performed the quantitative PLR was blinded to standard PLR and electrophysiological tests.

\section{Withdrawal of Life Support}

The objective of this study was to evaluate the prognostic value of automated pupillometry in comatose CA patients and to compare it to that of standard tools that were utilized routinely when assessing prognosis. According to our practice, neurological examination and EEG were performed at two time points, during TH and at about $48 \mathrm{~h}$, as described in our previous studies [7, 20, 23]. Additional tests were performed at 48 and $72 \mathrm{~h}$, including SSEP and NSE. The decision to withdraw life support was based on a multimodal approach that included all these tests, and the final decision was taken following a consensus between neurologists and intensivists in charge of the patient. Withdrawal of life support was never done before $72 \mathrm{~h}$, to avoid premature withdrawal. Specifically, withdrawal of life support was considered upon the occurrence of two or more out of four criteria after more than $72 \mathrm{~h}$ after CA: incomplete return of brainstem reflexes, treatment-resistant myoclonus, non-reactive EEG background, bilateral absence of N20 on SSEP. Importantly, the results of automated quantitative pupillometry were not taken into account for the decision of withdrawal of life support.

\section{Data Collection and Outcome Assessment}

Baseline demographic data included age, gender, initial arrest rhythm (shockable $=$ ventricular fibrillation vs. non shockable $=$ asystole or pulseless electrical activity), and time to return of spontaneous circulation (ROSC). Outcome was assessed at 3 months by a semi-structured telephone interview with the patient or the patient's relatives using the GlasgowPittsburgh Cerebral Performance Categories (CPC) [24].

\section{Statistical Analysis}

For the present study, the percentage of pupillary response to a calibrated light stimulation measured with an automated infrared pupillometry was considered for the analysis. Associations of quantitative PLR with 90-day outcome (dichotomized as good $=$ CPC $1-2$, including good recovery and moderate disability, vs. poor $=$ CPC $3-5$, including severe disability, vegetative state and death) were analyzed using non-parametric Wilcoxon test. Quantitative PLR values were then assessed for sensitivity and specificity to identify the best cut-off value for the prediction of 90-day poor outcome at both time points studied, i.e., day 1 after $\mathrm{CA}$ during $\mathrm{TH}$ and sedation, and day 2 after $\mathrm{CA}$, following $\mathrm{TH}$ and rewarming, in normothermia, off sedation. Prognostic variables (including: presence/absence of standard PLR, performed simultaneously to quantitative PLR, at both time points; the best value of quantitative PLR, expressed as the \% pupillary response, at both time points; presence/absence of EEG background reactivity at day 2; presence/absence (bilaterally) of N20 on SSEP at day 2) were assessed for poor outcome prediction using the area under the receiver operating characteristic (ROC) curve. Multivariable logistic regression was also performed to adjust outcome prediction of quantitative PLR for known prognostic predictors (time from CA to ROSC and initial arrest rhythm). The results of the logistic regression were expressed as the odds ratio and $95 \%$ confidence intervals of poor 90-day outcome for each $10 \%$ increase of pupillary response 
assessed with automated pupillometry. Comparisons between the ROC curves were analyzed using $\chi^{2}$ test. All analyses were conducted using STATA 12 (STATA ${ }^{\circledR}$ Corporation, College Station, TX, USA). Statistical significance was set at $p<0.05$.

\section{Results}

\section{Patient Characteristics}

From June 2012 to June 2013, 50 consecutive comatose patients (16 women and 34 men) resuscitated from out-ofhospital CA were included in this study. All patients were treated with $\mathrm{TH}$ and no patients were excluded. Patient characteristics are summarized in Table 1. At 3 months, 23 (46\%) patients survived and had a good neurological recovery (CPC 1-2) vs. 27 (54\%) patients who had a poor outcome. Among patients with poor outcome, no subject had a CPC 3 (severe disability) or 4 (vegetative state) therefore all patients had a CPC 5 (death). Average time to death was 5 days: all subjects died from withdrawal of life support due to severe hypoxic-ischemic encephalopathy, and all deaths occurred in-hospital (during the ICU stay for the majority of patients).

Quantitative Pupillometry in Comatose Cardiac Arrest Patients

Across all patients, quantitative PLR was lower at day 1 , during $\mathrm{TH}$ and sedation-analgesia [median $14 \%$ (range 1-30\%)], than at day 2 , in normothermia, off sedationanalgesia [16\% (range 1-55\%), $p=0.005$ ].

Table 1 Patient baseline demographics

\begin{tabular}{ll}
\hline Variable & Value \\
\hline Patients number & 50 \\
Age (years) & $61(31-88)$ \\
Gender, female/male & $16 / 34$ \\
Time to ROSC (min) & $20(5-50)$ \\
Initial arrest rhythm & \\
Shockable (ventricular fibrillation) & 30 \\
Non-shockable (asystole + pulseless electrical & 20 \\
activity) & $(10+10)$ \\
Cerebral performance categories at 3 months & 10 \\
1. Good recovery & 13 \\
2. Moderate disability & 0 \\
3. Severe disability & 0 \\
4. Vegetative state & 27 \\
5. Death &
\end{tabular}

Data are presented as median (minimum-maximum)

ROSC return of spontaneous circulation
When looking at the two outcome groups, baseline pupil size measured with the automated pupillometer did not differ between patients with good outcome and those with poor outcome, both during TH [on average $16 \mathrm{~h}$ from CA: $2.2 \mathrm{~mm}$ (range $1.4-4.3 \mathrm{~mm}$ ) vs. $2.2 \mathrm{~mm}$ (range $1.7-5.3 \mathrm{~mm}), p=0.53$ ] and after passive rewarming in normothermia [on average $46 \mathrm{~h}$ from CA: $2.3 \mathrm{~mm}$ (range $1.2-4.4 \mathrm{~mm}$ ) vs. $2.2 \mathrm{~mm}$ (range $1.2-5.6 \mathrm{~mm}$ ), $p=0.91]$.

Quantitative PLR was strongly associated with 3-month outcome, when performed at the two time points (Table 2). Quantitative PLR was first measured at day 1 during TH, under sedation and analgesia: patients with good outcome had a median quantitative PLR of $16(9-23) \%$ versus 10 $(1-30) \%$ in the poor outcome group $(p=0.0005)$. The second test performed at day 2 after rewarming and off sedation/analgesia was similarly highly predictive of outcome: median quantitative PLR was 20 (13-39) \% among patients with good outcome versus $11(1-55) \%$ in the poor outcome group ( $p=0.0001)$. Figure 1 illustrates ranges of individual $\%$ pupillary responses for the two outcome groups at the two time points, at day 1 , during TH (A) and at day 2, after rewarming, at normothermia (B).

Of note, the total daily dose of sedatives (midazolam) and vasopressors (norepinephrine) did not differ significantly between the two outcome groups. The median fentanyl dose differed significantly between the two outcome groups, but was actually higher in patients with good versus poor outcome $(1.8$ vs. $1.1 \mathrm{mg} /$ day, $p=0.03$; Table 2). No patient received paralytics during the tests.

Quantitative Versus Standard Pupillary Reactivity to Predict 90-Day Outcome

Using area under the ROC curve analysis, the best cut-off value for quantitative PLR to predict 90-day poor outcome was found $<13 \%$ (Fig. 2), at both time points, with an area under the ROC curve 0.79 [95\% confidence intervals (CI) $0.68-0.90$ ] at day 1 during $\mathrm{TH}$ and $0.81(95 \% \mathrm{CI}$ $0.72-0.91)$ at day 2.

A quantitative PLR $<13 \%$ had significantly higher accuracy to predict 90-day poor outcome than absence of standard PLR, both at day 1 (area under the ROC curve 0.79 vs. $0.56, p=0.005$ ) and at day 2 (area under the ROC curve 0.81 vs. $0.64, p=0.006$, Fig. 3 ).

\section{Prognostic Accuracy of Quantitative PLR Versus EEG} and SSEP

At day 2, in normothermic conditions and off sedationanalgesia, the ROC area of quantitative PLR $<13 \%$ to predict 90-day poor neurological outcome was 0.81 and did not differ significantly from that of absent EEG reactivity and bilaterally absent N20 on SSEP (tested on average 
Table 2 Univariate associations with 90-day neurological outcome

\begin{tabular}{|c|c|c|c|}
\hline Variable & $\begin{array}{l}\text { Good outcome } \\
\text { (CPC 1-2) }\end{array}$ & $\begin{array}{l}\text { Poor outcome } \\
\text { (CPC 3-5) }\end{array}$ & $p$ \\
\hline \multicolumn{4}{|l|}{ Non-neurological variables } \\
\hline Age (years) & $60(34-81)$ & $63(31-88)$ & 0.096 \\
\hline Time from CA to return of spontaneous circulation (min) & $15(5-30)$ & $25(5-50)$ & $<0.0001$ \\
\hline Initial arrest non-shockable rhythm (nr/total nr) & $4 / 23$ & $16 / 27$ & 0.0026 \\
\hline Total daily midazolam dose (mg) & $161(0-361.2)$ & $125.6(0-257.3)$ & 0.11 \\
\hline Total daily fentanyl dose $(\mu \mathrm{g})$ & $1,775(0-5,245)$ & $1,072(0-3,373)$ & 0.033 \\
\hline Total daily dose norepinephrine (mg) & $5.1(0-44)$ & $6.2(0-177.7)$ & 0.76 \\
\hline \multicolumn{4}{|l|}{ Neurological variables } \\
\hline Absent standard PLR at day 1 after CA (during $\mathrm{TH}$ ) (nr/total nr) & $5 / 23$ & $9 / 27$ & 0.36 \\
\hline Quantitative PLR at day 1 after CA (during TH) (\%)* & $16(9-23)$ & $10(1-30)$ & 0.0005 \\
\hline Absent standard PLR at day 2 after CA (nr/total nr) & $1 / 23$ & $9 / 27$ & 0.01 \\
\hline Quantitative PLR at day 2 after CA (\%)* & $20(13-39)$ & $11(1-55)$ & 0.0001 \\
\hline Unreactive EEG background at day 2 after CA (nr/total nr) & $0 / 23$ & $15 / 25$ & $<0.0001$ \\
\hline Bilaterally absent N20 on SSEP at day 2 after CA (nr/total nr) & $0 / 23$ & $10 / 22$ & 0.0002 \\
\hline
\end{tabular}

Data are presented as median (ranges)

$C A$ cardiac arrest, $C P C$ cerebral performance categories, $E E G$ electroencephalography, SSEP somato-sensory evoked potentials, $T H$ therapeutic hypothermia

* Quantitative pupillary light reactivity [PLR, expressed as the percentage response of pupillary reactivity to a calibrated light stimulus (320 Lux, $1 \mathrm{~s})$ ] was measured with an automated infrared pupillometry: the best value of triplicates performed on both eyes was retained for the analysis

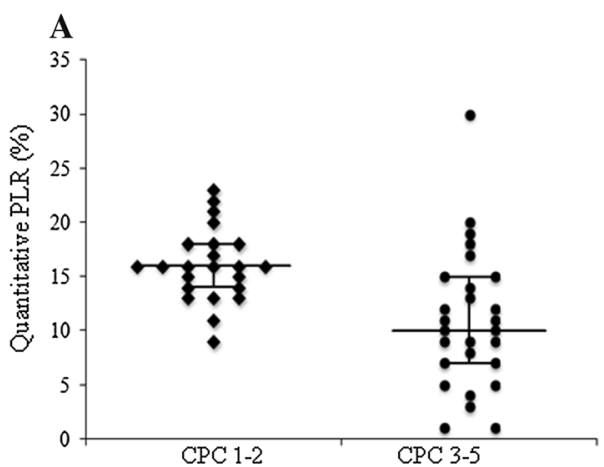

Fig. 1 Individual percentage of pupillary light response in the good outcome (CPC 1 and 2) and the poor outcome (CPC 3-5) group at day 1 (during therapeutic hypothermia and sedation, panel $A$ ) and at day 2

$14 \pm 11 \mathrm{~h}$ after rewarming: area under the ROC curve 0.81 for quantitative PLR $<13 \%$ vs. 0.80 for absent EEG reactivity and 0.73 for bilaterally absent SSEP, respectively, both $p>0.20, \chi^{2}$ test), thereby suggesting comparable prognostic accuracy of quantitative pupillometry when compared to electro-physiological tests (Table 3).

\section{Quantitative PLR is an Independent Predictor of 90-day}

Outcome

Longer time to ROSC and an initial non-shockable CA rhythm (asystole of pulseless electrical activity) were both associated with poor neurological outcome (Table 2). After adjustment for time to ROSC and initial CA rhythm, the

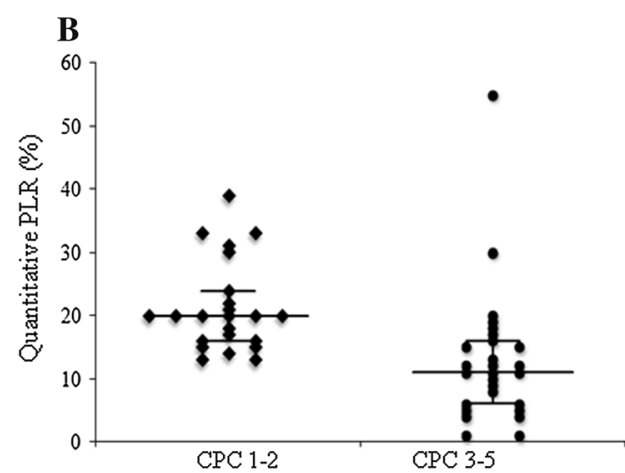

(after rewarming, at normothermia and off sedation, panel B). The large line represents the median, the small lines the interquartile ranges. $C P C$ cerebral performance categories

percentage pupillary response to a calibrated light stimulation measured with automated pupillometry at day 2 remained a strong independent predictor of outcome: a $10 \%$ increase of quantitative PLR was associated with a $71 \%$ reduction in the risk of poor neurological recovery at 90 days (adjusted odds ratio 0.29, confidence intervals $0.10-0.84, p=0.023$, Table 4).

\section{Discussion}

The findings of our study can be summarized as follows: (1) we show for the first time that quantitative pupillary reactivity using automated infrared pupillometry was 
Fig. 2 ROC curve analysis for quantitative pupillary light reactivity (PLR), defined as the percentage pupillary response to a calibrated light stimulus (320 Lux, 1 s). A quantitative PLR $<13 \%$ was the best cutoff value to predict 90 -day poor neurological outcome

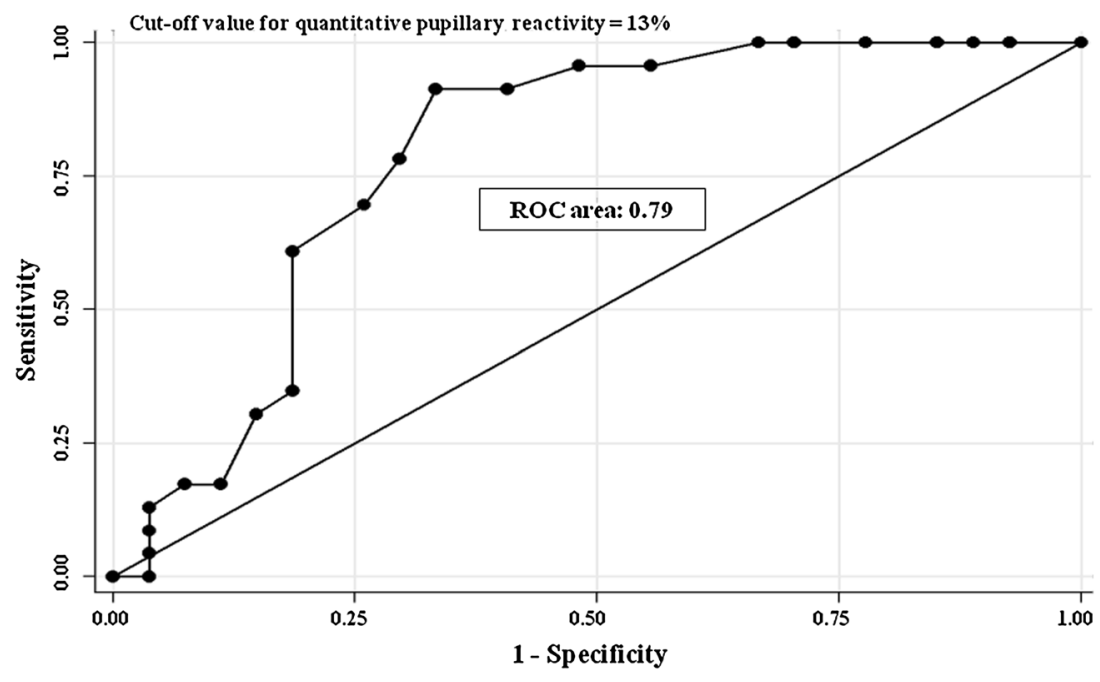

superior to standard qualitative pupillary reactivity using a manual pen light in predicting long-term neurological recovery following post-CA coma, both during $\mathrm{TH}$ and sedation and in normothermic conditions without sedationanalgesia; (2) the percentage increase in pupillary reactivity was a strong predictor of prognosis, independent of the duration of CA and the initial arrest rhythm; (3) in our single-center cohort, quantitative pupillary reactivity had comparable prognostic performance than electro-physiological exams, including EEG background reactivity and SSEP.

Neurological Examination for the Prognostication of Coma After CA

Before $\mathrm{TH}$ was considered a standard of care for the treatment of patients with coma after CA, the absence of motor response and the lack of pupillary and corneal reflexes were considered strong predictors of poor outcome, with a false-positive rate for poor prognosis close to $0 \%$ at day 3 [4]. However, following the increasing utilization of $\mathrm{TH}$, several studies demonstrated that neurological examination-although still remaining a key step in the evaluation of prognosis-may be inaccurate, partly because of the confounding effect of hypothermia and sedation on neurological responses $[5,6]$. This may lead to premature false diagnoses of poor prognosis in patients who may eventually awake, as recently shown by Perman et al. [25] who found that among 28 patients with early clinical signs of "poor" neurological prognosis, six eventually survived to discharge with favorable neurologic recovery. Standard PLR may be misleading [26, 27] and it has been recommended to complement it by other prognostic tools, such as EEG and SSEP [28]. Although of great value, the disadvantage of all electrophysiological tests is that they are not quantitative and require specific expertise and substantial ICU implementation.

\section{Quantitative Versus Standard Pupillary Light Response}

Automated pupillometry has the advantage to be quantitative, simple to use and therefore potentially widely available and easy to implement in routine critical care practice. Here we showed that quantitative PLR is superior to standard PLR in predicting neurological outcome of post-CA coma. Despite the \% pupillary response to a calibrated light stimulus was lower during $\mathrm{TH}$ and sedation than in normothermic conditions and off sedation/analgesia, the prognostic value of quantitative PLR was not affected by sedation, analgesia, or the amount of vasopressors. This reinforce the validity of automated pupillometry in providing precise measurement of pupil size and reactivity to light stimulation, irrespective of temperature, sedation, and vasopressor conditions, already at an early phase (within $48 \mathrm{~h}$ ) following coma after CA.

Automated Pupillometry has Comparable Prognostic Accuracy than Electro-physiological Exams

Because EEG and SSEP are part of standard care at our center and are increasingly used to improve the accuracy of coma prognostication after CA, we also analyzed whether the prognostic accuracy of quantitative PLR was at least comparable to that of electro-pysiological exams. By comparing the area under the ROC curve to predict 90-day outcome of the 3 tests, we found that indeed quantitative PLR yielded comparable prognostic accuracy than EEG reactivity and SSEP. As we previously said, electrophysiological tests are of great value to improve prognostic accuracy of coma after CA. However, EEG and SSEP are 
Fig. 3 Areas under the ROC curve for automated quantitative pupillary light reactivity (PLR, cut-off $<13 \%$ ) and standard PLR to predict 90-day poor neurological outcome, at day 1 after cardiac arrest, during therapeutic hypothermia and sedation (panel $A$ ) and at day 2 after cardiac arrest, following rewarming, in normothermia and off sedation (panel B). Quantitative PLR had better prognostic performance than standard PLR at day 1 ( 0.79 vs. $0.56, p=0.005)$ and day 2 (0.81 vs. $0.64, p=0.006)$
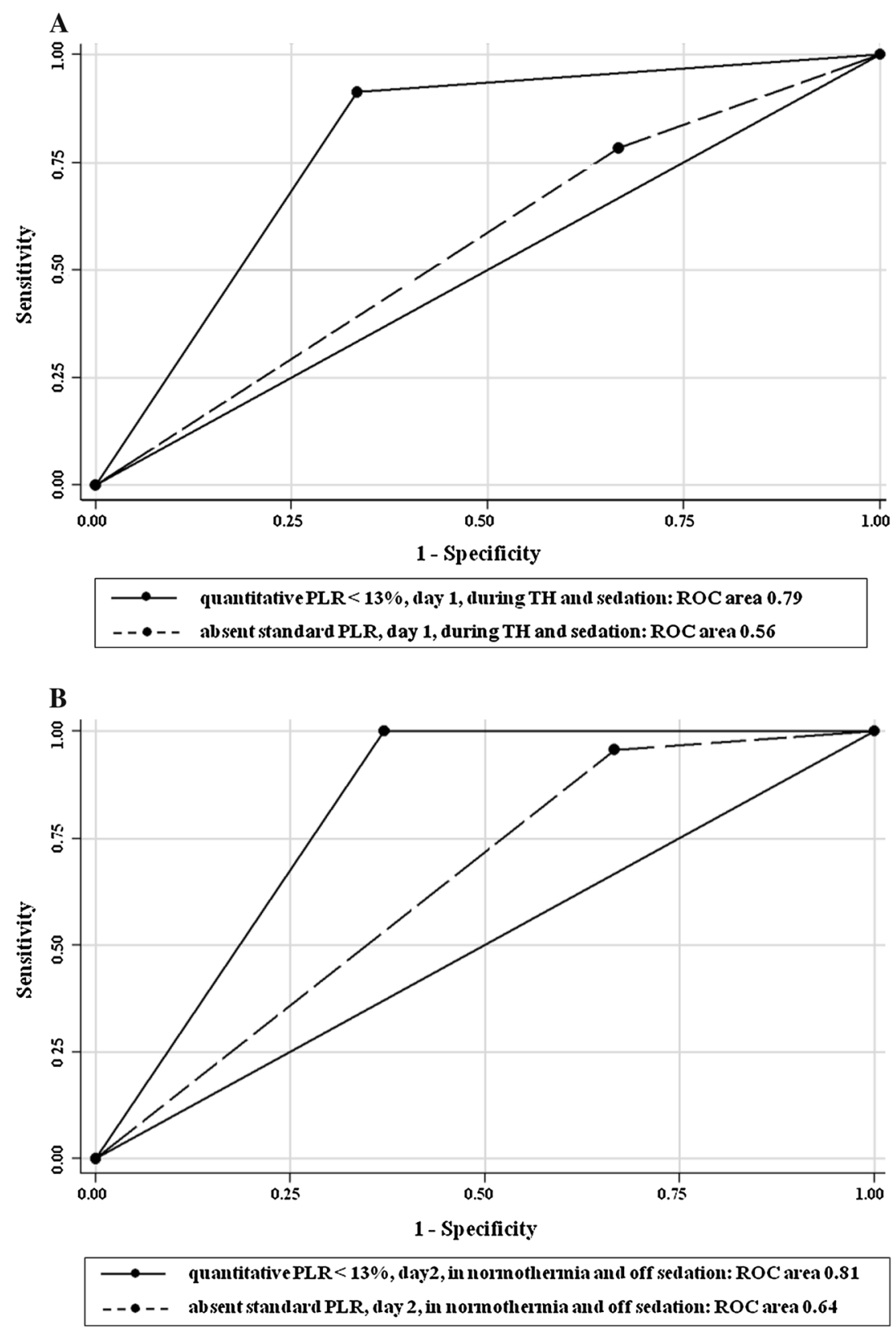

not always available and require both a specific expertise and substantial effort for ICU implementation.

In summary, our findings identify quantitative automated pupillometry as a new method of coma prognostication, which seems unaffected by TH and drug elimination, appears more accurate than standard pupillary light response and seems to have comparable prognostic performance than electro-physiological exams.

Our results are promising and prompt further larger multicenter studies to confirm our findings and to evaluate the value of quantitative pupillometry for the prognostication of post-CA coma. Awaiting such confirmatory studies, prognostication of CA should rely on a multimodal approach, including clinical examination, EEG, SSEP, and serum biomarkers (such as neuron specific enolase) [15, 20, 29]. It is conceivable that quantitative pupillometry may in the future be incorporated into such multimodal prognostic approach and become a standard tool of coma prognostication.

\section{Study Limitations}

The main limitation of this study is the sample size and the fact that it was single-center therefore data may not be generalized. However, data come from a homogeneous 
Table 3 Prognostic performance for 90-day neurological outcome of standard pupillary reactivity, quantitative pupillary reactivity, electroencephalography (EEG), and somato-sensory evoked potentials (SSEP), assessed using the area under the ROC curve analysis

\begin{tabular}{|c|c|c|c|c|c|}
\hline Variable & Sensitivity $(\%)$ & Specificity (\%) & PPV $(\%)$ & NPV $(\%)$ & $\mathrm{ROC}$ area \\
\hline \multicolumn{6}{|c|}{ Day 1 after cardiac arrest (during therapeutic hypothermia, under sedation-analgesia) } \\
\hline Quantitative PLR $<13 \% *$ & $66.7(46-83.5)$ & $91.3(72-98.9)$ & $90(68.3-98.8)$ & $70(50.6-85.3)$ & $0.79(0.68-0.90)$ \\
\hline Absent standard PLR & $33.3(16.5-54)$ & $78.3(56.3-92.5)$ & $64.3(35.1-87.2)$ & $50(32.9-67.1)$ & $0.56(0.43-0.68)$ \\
\hline \multicolumn{6}{|c|}{ Day 2 after cardiac arrest (after rewarming, in normothermia, off sedation-analgesia) } \\
\hline Quantitative PLR $<13 \% *$ & $63(42.4-80.6)$ & $100(85.2-100)$ & $100(80.5-100)$ & $69.7(51.3-84.4)$ & $0.81(0.72-0.91)$ \\
\hline Absent standard PLR & $33.3(16.5-54)$ & 95.7 (78.1-99.9) & $90(55.5-99.7)$ & $55(38.5-70.7)$ & $0.64(0.54-0.74)$ \\
\hline Absent EEG reactivity & $60(38.7-78.9)$ & $100(85.2-100)$ & $100(78.2-100)$ & $69.7(51.3-84.4)$ & $0.80(0.70-0.90)$ \\
\hline Bilaterally absent SSEP & $45.5(24.4-67.8)$ & $100(85.2-100)$ & $100(69.2-100)$ & $65.7(47.8-80.9)$ & $0.73(0.62-0.83)$ \\
\hline
\end{tabular}

Data are presented as values (95\% confidence intervals)

$N P V$ negative predictive value, $P P V$ positive predictive value

* Quantitative pupillary light reactivity [PLR, expressed as the percentage response of pupillary reactivity to a calibrated light stimulus (320 Lux,

$1 \mathrm{~s})$ ] was measured with an automated infrared pupillometry. Standard PLR was measured with a manual pen light

Table 4 Multivariable logistic regression

\begin{tabular}{|c|c|c|c|}
\hline Variable & $\begin{array}{l}\text { Adjusted odds ratio (95\% } \\
\text { confidence intervals) } \\
\text { of unfavorable outcome (CPC 3-5) at } 90 \text { days }\end{array}$ & $z$ & $p$ value \\
\hline $10 \%$ increase in quantitative PLR at $48 \mathrm{~h}$ after cardiac arrest & $0.29(0.10-0.82)$ & -2.27 & 0.023 \\
\hline Time from CA to ROSC, min & $1.28(1.09-1.51)$ & 3.03 & 0.002 \\
\hline Non-shockable rhythm & $48.5(3.35-702.03)$ & 2.85 & 0.004 \\
\hline
\end{tabular}

Quantitative pupillary light reactivity [PLR, expressed as the percentage response of pupillary reactivity to a calibrated light stimulus (320 Lux, $1 \mathrm{~s})$ ] was measured with an automated infrared pupillometry. For a $10 \%$ increase of pupillary reactivity-measured by automated infrared pupillometry at day 2 in normothermia - there was a $71 \%$ reduction in the risk of poor neurological recovery at 90 days

$C A$ cardiac arrest, CPC cerebral performance categories, $R O S C$ return of spontaneous circulation

cohort of comatose patients with out-of-hospital CA treated with $\mathrm{TH}$, managed with a written standardized algorithm for induced cooling, sedation-analgesia, post-resuscitation care, neurological assessment and withdrawal of care, as described in our recent studies [7, 20-23]. In addition, robust statistical analysis was performed and comparisons with standard electrophysiological tests were conducted, which appear to reinforce the main findings of the study. While automated pupillometry provides a calibrated light stimulation, the standard pen light may give variable amount of illumination. This is a study bias and a major limitation, but on the other hand it reflects actual critical care practice more adequately.

An important issue to discuss is the relatively early prognostic assessment in our study. Automated pupillometry was in fact performed at a relatively early stage (within $48 \mathrm{~h}$ from $\mathrm{CA}$ ), according to the standard algorithm for the prognostication of coma after $\mathrm{CA}$ in use at our institution. However, we wish to underline that the objective of our study was not to approximate brain death at an early time point. Rather, the objective of the present investigation was to evaluate the prognostic value of a new tool for the quantitative measure of pupillary light response using an automated pupillometry in comatose CA patients and to compare it to that of standard pupil reactivity using a pen light. In our practice, neurological examination is performed in combination with EEG (performed at two time points, at day 1 , during $\mathrm{TH}$, and at day $2, \approx 48 \mathrm{~h}$ after CA) and SSEP, performed at day 2. The decision to withdraw life support is based on a multimodal approach that includes all these tests, and is taken after a consensus between neurologists and intensivists in charge of the patient, and never before $72 \mathrm{~h}$ [20]. All poor outcome patients died, so further study will be required to determine the accuracy of quantitative pupillometry to predict poor neurological recovery or death more specifically. Given the results of the recent targeted temperature management trial [30], and the likely shift to move from induced hypothermia to controlled normothermia in the near future, our results may need to be replicated under conditions of normothermia instead of hypothermia treatment. Finally, although the study was blinded for outcome assessments, we cannot completely exclude that quantitative pupillometry results may still have influenced treatment decisions and the so-called self-fulfilling prophecy inherent to this kind of study. 


\section{Conclusions}

Our single-center prospective cohort study shows that automated quantitative pupillometry is superior to standard pupillary examination in predicting poor 90-day outcome after CA, irrespective of hypothermic conditions and sedation and has comparable prognostic accuracy than electrophysiological tests, including EEG and SSEP. Our data suggest that quantitative pupillometry might be an accurate and simple tool for the prognostication of post-CA coma. Additional larger multicenter studies are warranted to confirm the value of quantitative pupillometry in this setting.

Acknowledgments The authors thank Helena Marti-Soler, Ph.D., for independent review of statistical analysis, and Christine Stähli, RN and Elsa Juan, MPsych, for her help in data acquisition. This work was supported by grants from the Swiss National Science Foundation (to MO and AOR), the European Critical Care Research Network (to MO), and the Gueules Cassées Foundation (to PB)

Conflict of interest The authors have no conflict of interest to declare. The manufacturer of automated pupillometry (IDMED) was not involved in the study.

\section{References}

1. Hypothermia after Cardiac Arrest Study Group. Mild therapeutic hypothermia to improve the neurologic outcome after cardiac arrest. N Engl J Med. 2002;346:549-56.

2. Bernard SA, Gray TW, Buist MD, et al. Treatment of comatose survivors of out-of-hospital cardiac arrest with induced hypothermia. N Engl J Med. 2002;346:557-63.

3. Kurz MC. For whom the bell tolls. Resuscitation. 2011;82:1371-2.

4. Wijdicks EF, Hijdra A, Young GB, Bassetti CL, Wiebe S. Practice parameter: prediction of outcome in comatose survivors after cardiopulmonary resuscitation (an evidence-based review): report of the Quality Standards Subcommittee of the American Academy of Neurology. Neurology. 2006;67:203-10.

5. Samaniego EA, Mlynash M, Caulfield AF, Eyngorn I, Wijman CA. Sedation confounds outcome prediction in cardiac arrest survivors treated with hypothermia. Neurocrit Care. 2011;15:113-9.

6. Tortorici MA, Kochanek PM, Poloyac SM. Effects of hypothermia on drug disposition, metabolism, and response: a focus of hypothermia-mediated alterations on the cytochrome P450 enzyme system. Crit Care Med. 2007;35:2196-204.

7. Rossetti AO, Oddo M, Logroscino G, Kaplan PW. Prognostication after cardiac arrest and hypothermia: a prospective study. Ann Neurol. 2010;67:301-7.

8. Al Thenayan E, Savard M, Sharpe M, Norton L, Young B. Predictors of poor neurologic outcome after induced mild hypothermia following cardiac arrest. Neurology. 2008;71:1535-7.

9. Fugate JE, Wijdicks EF, Mandrekar J, et al. Predictors of neurologic outcome in hypothermia after cardiac arrest. Ann Neurol. 2010;68:907-14.

10. Rossetti AO, Urbano LA, Delodder F, Kaplan PW, Oddo M. Prognostic value of continuous EEG monitoring during therapeutic hypothermia after cardiac arrest. Crit Care. 2010;14: R173.
11. Rundgren M, Westhall E, Cronberg T, Rosen I, Friberg H. Continuous amplitude-integrated electroencephalogram predicts outcome in hypothermia-treated cardiac arrest patients. Crit Care Med. 2010;38:1838-44.

12. Cloostermans MC, van Meulen FB, Eertman CJ, Hom HW, van Putten MJ. Continuous electroencephalography monitoring for early prediction of neurological outcome in postanoxic patients after cardiac arrest: a prospective cohort study. Crit Care Med. 2012;40:2867-75.

13. Bouwes A, Binnekade JM, Kuiper MA, et al. Prognosis of coma after therapeutic hypothermia: a prospective cohort study. Ann Neurol. 2012;71:206-12.

14. Kamps MJ, Horn J, Oddo M, et al. Prognostication of neurologic outcome in cardiac arrest patients after mild therapeutic hypothermia: a meta-analysis of the current literature. Intensiv Care Med. 2013;39:1671-82.

15. Oddo M, Rossetti AO. Predicting neurological outcome after cardiac arrest. Curr Opin Crit Care. 2011;17:254-9.

16. Larson MD, Muhiudeen I. Pupillometric analysis of the 'absent light reflex'. Arch Neurol. 1995;52:369-72.

17. Meeker M, Du R, Bacchetti P, et al. Pupil examination: validity and clinical utility of an automated pupillometer. J Neurosci Nurs. 2005;37:34-40.

18. Yan S, Tu Z, Lu W, et al. Clinical utility of an automated pupillometer for assessing and monitoring recipients of liver transplantation. Liver Transplant. 2009;15:1718-27.

19. Behrends M, Niemann CU, Larson MD. Infrared pupillometry to detect the light reflex during cardiopulmonary resuscitation: a case series. Resuscitation. 2012;83:1223-8.

20. Oddo M, Rossetti AO. Early multimodal outcome prediction after cardiac arrest in patients treated with hypothermia. Crit Care Med. 2014; Jan 22 (Epub ahead of print)

21. Engel H, Ben Hamouda N, Portmann K, et al. Serum procalcitonin as a marker of post-cardiac arrest syndrome and long-term neurological recovery, but not of early-onset infections, in comatose post-anoxic patients treated with therapeutic hypothermia. Resuscitation. 2013;84:776-81.

22. Cueni-Villoz N, Devigili A, Delodder F, et al. Increased blood glucose variability during therapeutic hypothermia and outcome after cardiac arrest. Crit Care Med. 2011;39:2225-31.

23. Rossetti AO, Carrera E, Oddo M. Early EEG correlates of neuronal injury after brain anoxia. Neurology. 2012;78:796-802.

24. Phelps R, Dumas F, Maynard C, Silver J, Rea T. Cerebral performance category and long-term prognosis following out-ofhospital cardiac arrest. Crit Care Med. 2013;41:1252-7.

25. Perman SM, Kirkpatrick JN, Reitsma AM, et al. Timing of neuroprognostication in postcardiac arrest therapeutic hypothermia. Crit Care Med. 2012;40:719-24.

26. Yannopoulos D, Kotsifas K, Aufderheide TP, Lurie KG. Cardiac arrest, mild therapeutic hypothermia, and unanticipated cerebral recovery. Neurologist. 2007;13:369-75.

27. Friberg H. Neurological prognostication after cardiac arrest. Scand J Trauma Resusc Emerg Med. 2008;16:10.

28. Sandroni C, Cavallaro F, Callaway CW, et al. Predictors of poor neurological outcome in adult comatose survivors of cardiac arrest: a systematic review and meta-analysis. Part 2: patients treated with therapeutic hypothermia. Resuscitation. 2013;84:1324-38.

29. Peberdy MA, Callaway CW, Neumar RW, et al. Part 9: postcardiac arrest care: 2010 American Heart Association Guidelines for Cardiopulmonary Resuscitation and Emergency Cardiovascular Care. Circulation. 2010;122:S768-86.

30. Nielsen N, Wetterslev J, Cronberg T, et al. Targeted temperature management at $33{ }^{\circ} \mathrm{C}$ versus $36{ }^{\circ} \mathrm{C}$ after cardiac arrest. N Engl J Med. 2013;369:2197-206. 